\title{
Effect of Self-etch Primer-adhesive and Conventional Adhesive Systems on the Shear Bond Strength and Bond Failure of Orthodontic Brackets: A Comparative Study
}

\author{
${ }^{1} \mathrm{~S}$ Sreedhara, ${ }^{2}$ Malthesh B Savakkanavar, ${ }^{3} \mathrm{RNG}$ Rajesh, ${ }^{4}$ Rajesh Kumar Reddy Ankireddy, ${ }^{5} \mathrm{~N}$ Sanjay, ${ }^{6} \mathrm{KS}$ Girish
}

\begin{abstract}
Background: Prompt-L-Pop is a sixth generation bonding system contains methacrylated phosphoric acid esters that combine an acidic component for etching the enamel and a primer, is an all-in-one adhesive. This study was undertaken to compare the bonding strength of brackets to enamel with traditional bonding technique and the new Prompt-L-Pop system using the same composite resin.
\end{abstract}

Materials and methods: In this in vitro experimental study, 60 human premolar teeth extracted for orthodontic treatment were collected. The samples were randomly divided into three groups comprising of 20 teeth in each group. Shear bond strength and ARI scores for the specimens were measured. Comparison was done using one way ANOVA and Chi-square test.

Results: Fourth generation bonding adhesive system depicted similar bond strength to fifth generation bonding adhesive system. Both fourth and fifth generation exhibited higher shear bond strength as compared to sixth generation bonding adhesive system.

Conclusion: Fourth and fifth generation exhibited higher shear bond strength as compared to sixth generation bonding adhesive system but the sixth generation has clinically acceptable shear bond strength. Also, it was found that sixth

\footnotetext{
${ }^{1}$ Department of Orthodontics and Dentofacial Orthopedics SJM Dental College and Hospital, Chitradurga, Karnataka India

${ }^{2}$ Department of Orthodontics and Dentofacial Orthopedics Sharavathi Dental College and Hospital, Shimoga, Karnataka India

${ }^{3}$ Department of Orthodontics and Dentofacial Orthopedics Sri Rajiv Gandhi Dental College and Hospital, Bengaluru Karnataka, India

${ }^{4}$ Department of Orthodontics and Dentofacial Orthopedics Rajiv Gandhi Institute of Medical Sciences, Kadapa, Andhra Pradesh, India

${ }^{5}$ Department of Orthodontics and Dentofacial Orthopedics Apollo White Dental; Sri Rajiv Gandhi Dental College and Hospital, Bengaluru, Karnataka, India

${ }^{6}$ Department of Orthodontics and Dentofacial Orthopedics, Magi Orthodontic Dental Care Centre, Bengaluru, Karnataka, India

Corresponding Author: S Sreedhara, Reader, Department of Orthodontics and Dentofacial Orthopedics, SJM Dental College and Hospital, Chitradurga 577501, Karnataka, India Phone: 09844358031, e-mail: dr_136rajesh@rediffmail.com
}

generation leaves less residual adhesive on the tooth after bracket removal.

Keywords: Adhesive system, Bond failure, Prompt-L-Pop, Shear bond strength.

How to cite this article: Sreedhara S, Savakkanavar MB, Rajesh RNG, Ankireddy RKR, Sanjay N, Girish KS. Effect of Self-etch Primer-adhesive and Conventional Adhesive Systems on the Shear Bond Strength and Bond Failure of Orthodontic Brackets: A Comparative Study. J Contemp Dent Pract 2015;16(2):130-134.

Source of support: Nil

Conflict of interest: None

\section{INTRODUCTION}

Conventional adhesive systems use three different agents an enamel conditioner, primer and an adhesive resin. A unique characteristic of some new bonding systems is the combination of conditioner and the primer to form a single solution for simultaneous use on both enamel and dentin. Combining conditioning and priming agents into a single treatment step ${ }^{1}$ results in reduced time and improved cost-effectiveness for the clinician and indirectly for the patient.

Acid-etch bonding technique is widely used in orthodontia for attaching brackets to the tooth. But, the clinician is always concerned as debonding leaves the enamel surface unesthetic and prone to caries. As enamel fracture and crazing is reported more often than debonding, particularly with ceramic brackets, as bond failure at the bracket-adhesive interface or within the adhesive is more desirable (safer) than at the adhesive-enamel interface. ${ }^{2}$ Hence, acidic primers and conditioners, like maleic acid, ${ }^{3}$ have been alternately tested to determine if they can attain a clinically acceptable orthodontic bracket bond strength while decreasing the depth of enamel dissolution and decreasing the number of steps during the bonding procedure.

The necessity to make bonding systems always more reliable, biocompatible and, most of all, easy to use and insensitive to saliva contamination has served as the 'driving force' for the development of materials that could etch, prime and bond in one step. ${ }^{4}$ Prompt-L-Pop (ESPE 
Dental AG, Seefeld, Germany), contains methacrylated phosphoric acid esters that combine an acidic component for etching the enamel and a primer, was introduced as an all-in-one adhesive for composites and compomers. It is claimed to provide good bonding characteristics for enamel and dentin. The Prompt-L-Pop despite its low $\mathrm{pH}$, produces an etching similar to phosphoric acid. Moreover, the simultaneous penetration of bonding and etching agents avoids technical mistakes. Evaporation of the solvent after the bottle is opened is avoided because the Prompt-L-Pop bonding system is composed of three sealed and segregated compartments. This style of packaging not only eliminates the problem of evaporation, but it also prevents cross-contamination.

Presumably, as a consequence of the neutralization of acid monomers, the $\mathrm{pH}$ of phosphoric esters rises as the etching progresses. The drop in hydrogen ion concentration halts demineralization. During the etching phase the hydrogen ions released by hydroxyapatite crystals are chelated in the primer. Thus, it is not necessary to rinse. Because penetration of bonding monomers and demineralization take place simultaneously, poor infiltration of the bonding material is not an issue. This monocomponent system, if truly dependable and practical, would mean a significant advantage in orthodontics compared with the traditional pluricomponent techniques. ${ }^{5}$

In a study by Bishara SE et al (2001), ${ }^{6}$ the use of this self-etch primer provided significantly lower but clinically acceptable shear bond strength when compared with that of phosphoric acid and a sealant, before bonding orthodontic brackets with a composite adhesive. In another study ${ }^{7}$ (Bishara SE et al, in preparation), an experimental self-etch primer (EXL \#547, 3M ESPE, St Paul, Minn) was evaluated and was found to provide similar shear bond strengths to those obtained with the conventional adhesive systems.

Prompt-L-Pop is a sixth generation bonding system presented in a disposable blister with a brush. Because all its components are sealed, it is also hygienic. It is a bicomponent hydrophilic adhesive. This study was undertaken to compare the bonding strength of brackets to enamel with traditional bonding technique and the new Prompt-L-Pop system using the same composite resin (Transbond XT, Unitek, Monrovia, CA, USA).

\section{MATERIALS AND METHODS}

In this in vitro experimental study, 60 human premolars which were extracted for orthodontic treatment were collected from the Department of Oral and Maxillofacial Surgery, College of Dental Sciences, Davangere. Sample size was calculated based on pilot study. Ethical clearance for the study was obtained from the institutional ethics committee. Tooth selection criteria included: Intact buccal enamel, tooth not subjected to any pretreatment chemical agents, e.g. $\mathrm{H}_{2} \mathrm{O}_{2}$, etc. with no cracks due to pressure of the forceps and no caries. Sixty preadjusted stainless steel brackets (Gemini, 3M Unitek, Monrovia, USA), with bracket base surface area $9 \mathrm{~mm}^{2}$ were used in the study (Fig. 1).

The samples were randomly divided into three groups comprising of 20 teeth in each group.

\section{Group I: 4th Generation (Scotchbond)}

Twenty teeth were etched with $37 \%$ phosphoric acid gel. The teeth were thoroughly washed and dried. Primer and adhesive liquid was applied. The brackets were then bonded with Scotchbond and light cured for 20 seconds according to the manufacturer's instructions.

\section{Group II: 5th Generation (Prime and Bond)}

Twenty teeth were etched with $37 \%$ phosphoric acid gel. The teeth were thoroughly washed and dried. Prime bond is applied and then air dried. The brackets were then bonded with Transbond XT and light cured for 20 seconds according to the manufacturer's instructions.

\section{Group III: 6th Generation (Prompt-L-pop)}

The self etch primer Prompt-L-Pop was placed on the enamel of 20 teeth for 15 seconds and gently evaporated with air, according to the manufacturer's instructions. For activation of the primer, the two components are squeezed together, and the resulting mix is applied directly on the tooth surface. The brackets were then bonded with Transbond XT and light cured for 20 seconds. All samples were stored in deionized water at $37^{\circ} \mathrm{C}$ for 48 hours.

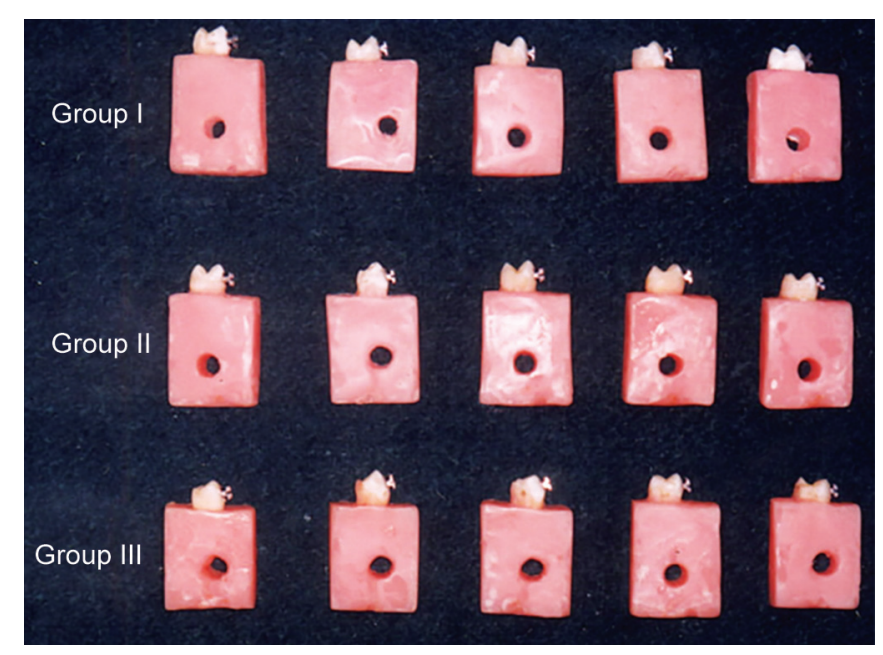

Fig. 1: Teeth mounted in acrylic blocks with brackets 


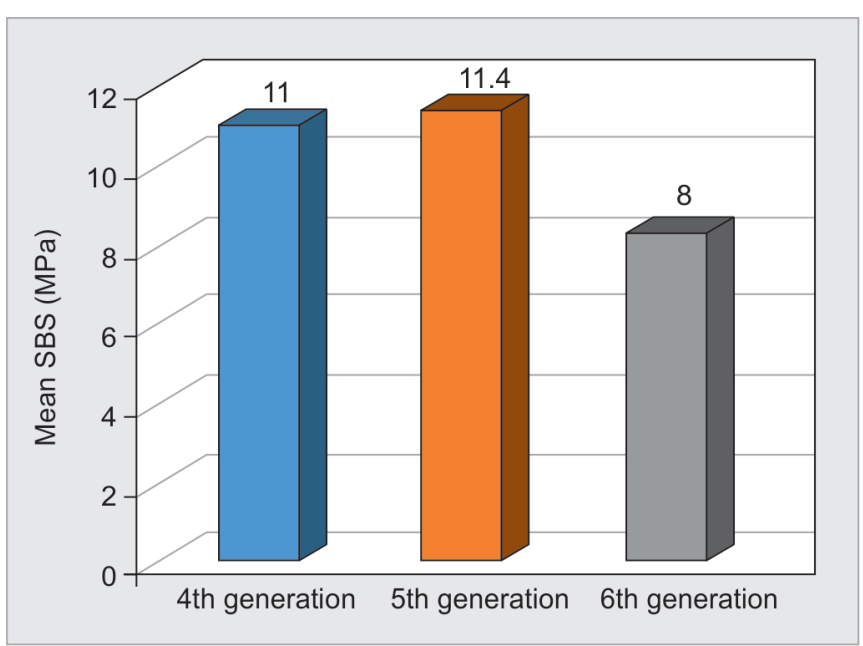

Graph 1: Comparison of shear bond strength (MPa) between 4th, 5th and 6th (prompt-L-PoP) generation bonding adhesive systems

\section{Shear Bond Strength Measurement}

Shear bond force was tested using Universal Testing Machine. A shear force at the bracket tooth interface was produced by applying an occlusogingival load on the bracket. A computer connected to the Universal testing machine recorded the results of each test. A crosshead speed of $5 \mathrm{~mm} /$ minute was considered for measuring shear bond strengths.

\section{Site of Bond Failure}

The teeth and brackets were examined with a stereomicroscope (at 10× magnification) after being debonded. The modified adhesive remnant index (ARI) was used to assess the amount of resin material that adhered to the enamel surface after removing the bracket (Fig. 2). The ARI scale has a range of 5 to 1 (score 5 -no composite remained on the enamel; score 4 -less than $10 \%$ of composite remained on the tooth surface; score 3 - more than $10 \%$ but less than $90 \%$ of the composite remained on the tooth; score $2-$ more than $90 \%$ of the composite remained; score 1-all of the composite, with an impression of the bracket base, remained on the enamel surface).

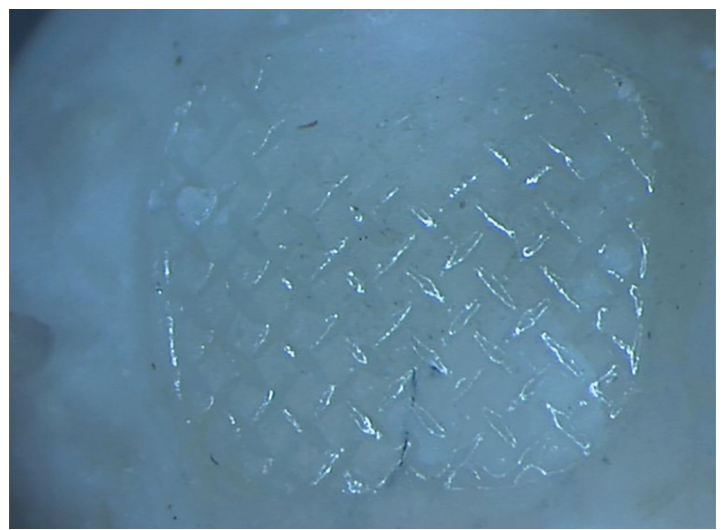

Fig. 2: Stereomicroscopic picture of tooth surface to determine ARI

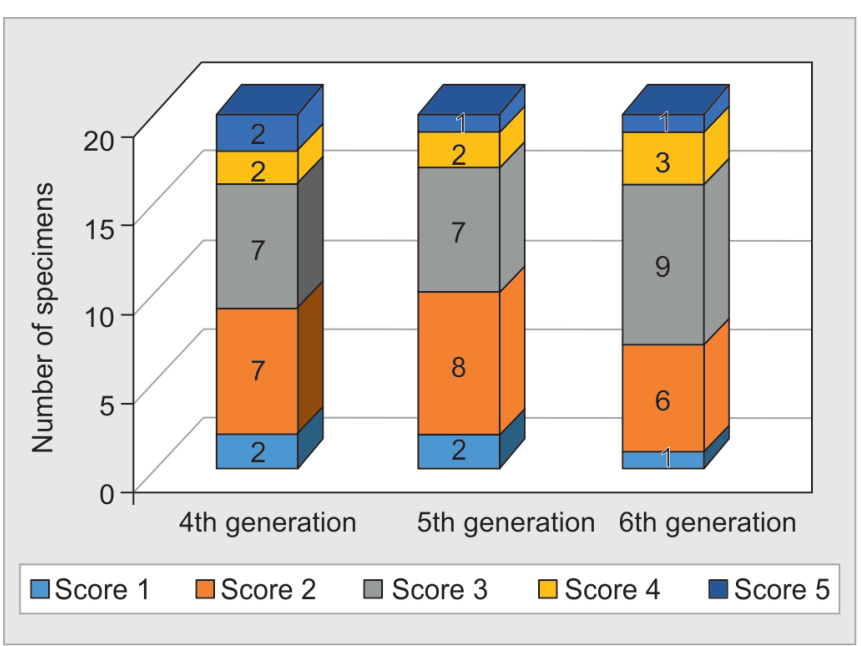

Graph 2: Comparison of ARI scores in 4th, 5th and 6th (prompt-L-PoP) generation bonding adhesive systems

\section{STATISTICAL ANALYSIS}

As the data were continuous in nature, range, mean and standard deviation were calculated, and parametric tests were used for analysis. Intergroup comparison was performed by one way analysis of variance followed by pairwise comparison using student's t-test. The pattern of ARI scores between three groups was compared using Chi-square test. Statistical significance was considered at less than 0.05 .

\section{RESULTS}

The shear bond strengths were recorded in Kgf and converted into MPa. In each group, mean and standard deviation were calculated as illustrated in Table 1.

Intergroup comparison of the bond strengths was done by one way analysis of variance (ANOVA) followed by student's t-test for pairwise comparison as illustrated in Table 2.

One way ANOVA revealed that group I-fourth generation bonding adhesive system (mean $11.04 \pm 1.42$ $\mathrm{MPa}$ ) and group II-fifth generation bonding adhesive system (mean $11.4 \pm 0.9 \mathrm{MPa}$ ) exhibited almost same bond strengths. Group III-6th generation (Prompt-LPop) bonding adhesive system (mean $8.03 \pm 1.44$ ) showed less shear bond strength as compared to groups I and II (Graph 1).

Table 1: Mean shear bond strength in the study groups

\begin{tabular}{|c|c|c|c|c|c|}
\hline \multirow{2}{*}{$\begin{array}{l}\text { S. } \\
\text { no. }\end{array}$} & \multirow[b]{2}{*}{ Study groups } & \multicolumn{2}{|c|}{ Kgf } & \multicolumn{2}{|c|}{$\mathrm{MPa}$} \\
\hline & & Mean & $S D$ & Mean & $S D$ \\
\hline I & 4th generation & 10.13 & 1.30 & 11.04 & 1.42 \\
\hline II & $\begin{array}{l}\text { 5th generation } \\
\text { (conventional) }\end{array}$ & 10.83 & 0.85 & 11.40 & 0.99 \\
\hline III & $\begin{array}{l}\text { 6th generation } \\
\text { (L-Pop) }\end{array}$ & 7.37 & 1.32 & 8.03 & 1.44 \\
\hline
\end{tabular}


Effect of Self-etch Primer-adhesive and Conventional Adhesive Systems on the Shear Bond Strength

Table 2: Comparison of mean shear bond strength in the study groups using ANOVA followed by t-test

\begin{tabular}{|c|c|c|c|c|c|c|c|}
\hline \multirow[b]{2}{*}{ Groups } & \multirow[b]{2}{*}{$N$} & \multicolumn{3}{|c|}{ Shear bond strength (MPa) } & \multirow{2}{*}{$\begin{array}{l}\text { Groups } \\
\text { compared }\end{array}$} & \multirow[b]{2}{*}{$t$-value* } & \multirow[b]{2}{*}{$p$-value } \\
\hline & & Range & Mean & $S D$ & & & \\
\hline 4th generation & 20 & $8.8-13.3$ & 11.04 & 1.42 & $I-I I$ & 0.92 & $0.36 \mathrm{NS}$ \\
\hline 5th generation (conventional) & 20 & $9.5-13.4$ & 11.40 & 1.00 & $|-|||$ & 6.65 & $<0.001 \mathrm{HS}$ \\
\hline 6th generation (L-POP) & 20 & $5.6-10.8$ & 8.03 & 1.44 & II-III & 8.59 & $<0.001 \mathrm{HS}$ \\
\hline
\end{tabular}

One-way ANOVA (F: 40.4; $p<0.001$; HS: Highly significant; NS: Nonsignificant)

Table 3: Distribution of ARI in three groups

\begin{tabular}{|c|c|c|c|c|c|c|c|c|}
\hline \multirow[b]{2}{*}{ Groups } & \multirow[b]{2}{*}{ No. of cases } & \multicolumn{5}{|c|}{ ARI scores } & \multirow[b]{2}{*}{$\chi^{2}$} & \multirow[b]{2}{*}{$p$-value } \\
\hline & & 1 & 2 & 3 & 4 & 5 & & \\
\hline I. 4th generation & 20 & 2 & 7 & 7 & 2 & 2 & 1.82 & $0.99 \mathrm{NS}$ \\
\hline II. 5th generation & 20 & 2 & 8 & 7 & 2 & 1 & & \\
\hline III. 6th generation & 20 & 1 & 6 & 9 & 3 & 1 & & \\
\hline
\end{tabular}

NS: Nonsignificant

\section{ARI SCORES (GRAPH 2)}

Less residual adhesive remaining on the tooth was observed when the sixth generation bonding adhesive system was used (ARI score of 3 ) as shown in Table 3.

\section{DISCUSSION}

Traditionally, the use of acid etchants followed by a primer has been fundamental to the bonding procedure of composite adhesives to permit good wetting and penetration of the sealant into the tooth surface. The utilization of the new self etching primers is of the opinion to improve the manipulation of adhesive systems. ${ }^{8}$ The early acidic primers were selectively compatible with certain adhesives and, as a result, either produced significantly lower bond strength or needed significantly more working time.

\section{Development of a New Adhesion Concept ${ }^{9}$ (Prompt-L-Pop)}

In 1997, the R and D department at ESPE started a new concept for the adhesive dentistry, taking into consideration the following requirements:

- Reliable bonding quality

- Easy to use

- Compatible with all restorative materials

- Time saving

The overall goal for this concept was to make the dentist's work much easier and at the same time to keep the quality level, that is already known, from the total etch technique. It took a lot of effort and many screening experiments until the first solution was developed which at that time was referred to as 'pretreatment solution'. More in vitro and in vivo testing results finally lead to the all-in-one concept which resulted in the product Prompt-L-Pop which was introduced into the market in April 1999. This new adhesion concept also included a new and innovative packaging of the product in a blister foil that contains three compartments.

- Compartment one: Methacrylated phosphoric acid esters, photoinitiators and stabilizers.

- Compartment two: Water, complex fluoride and stabilizers.

- Compartment three: Microbrush.

The blister is activated by squeezing compartment one, thereby releasing its content into compartment two. The mixing ratio is $4: 1$ and the freshly mixed solution is released on the microbrush into compartment three by squeezing compartment two.

\section{Prompt-L-Pop-How does It work?}

The rationale behind Prompt-L-Pop is that when rubbed on the enamel for 15 seconds, it would dissolve the smear layer as well as etch the enamel surface to create the hydroxyapatite etching pattern. By air-drying a very thin film of Prompt-L-Pop is formed on top of the enamel which enables the micromechanical retention of a restorative material in combination with a chemical bond between this thin Prompt-L-Pop layer and the composite material.

The major benefit of this new concept for orthodontist is that they will always work with the freshly mixed solution in combination with an extremely simplified working procedure. ${ }^{10}$ Using Prompt-L-Pop takes only three steps until the first increment of restorative material is placed compared to seven steps for a fifth generation and ten steps for fourth generation adhesive material.

\section{In vitro and in vivo Results ${ }^{9}$}

Before this new adhesion concept was introduced into the market, the quality of Prompt-L-Pop was tested intensively, 21 in vitro studies and nine in vitro studies were performed. The results of the in vitro studies are very promising. It showed that with Prompt-L-Pop the 
same or even better results were obtained although no separate etching step was necessary.

Scanning electron microscopy (SEM) studies $^{11}$ revealed that the etching pattern produced by PromptL-Pop on enamel is more or less the same as that of phosphoric acid etching. For the adhesion to dentin it was shown by confocal laser scanning microscopic studies that with Prompt-L-Pop a hybrid layer in dentin was formed. Regarding marginal integrity, SEM studies indicated that Prompt-L-Pop produced equal or even better results than other systems like Prime and Bond.

Data collected from the in vivo studies so far, indicate that with Prompt-L-Pop good clinical results are obtained in combination with a significant reduction in post-operative sensitivity. This might be due to the fact that Prompt-L-Pop demineralization and resin penetration of dentin appears at the same time in just one step. Therefore, the risk of nanoleakage is reduced significantly resulting in less postoperative sensitivity.

Several investigators have evaluated the bond strengths of self-etching primers. A study by Bishara et $\mathrm{al}^{3}$ found that acidic primers can produce clinically acceptable shear bond strengths of $10 \mathrm{MPa}$ when used with highly filled composites. In another study, however, Bishara and colleagues ${ }^{12,13}$ found bond strengths to be inadequate when the acidic primer Clearfil Liner Bond 2 was used with Transbond XT composite resin.

Rueggeberg et $\mathrm{al}^{14}$ determined that Prompt-L-Pop without acid etching produced similar bond strengths as with conventional bracket placement techniques. Hitmi ${ }^{15}$ found no significant difference between Prompt-L-Pop and a control adhesive, which used a $37 \%$ phosphoric acid etchant. Bergeron et $\mathrm{al}^{16}$ concluded that the resin enamel bond strength of seven different self-etching primers, including Prompt-L-Pop, was similar to or better than that of multiple step systems. In a recent study, Bishara et $\mathrm{al}^{4,17,18}$ showed that a self-etching primer produced a significantly lower, but clinically acceptable, shear bond strength compared to acid etching when used with Transbond XT composite resin.

There was a tendency to have less residual adhesive remaining on the tooth when Prompt-L-Pop was used (ARI scores of 3). This might be of advantage to the clinician because it will require less time to clean the teeth after debonding, in addition, to the fact that the enamel etchant and the primer are placed in one step.

\section{CONCLUSION}

Following conclusions were made from the present study:

1. Fifth generation (Prime and Bond) bonding agent has highest shear bond strength followed by fourth (Scotchbond) and sixth generation (Prompt-L-pop).
2. Fourth and fifth generation exhibited higher shear bond strength as compared to sixth generation bonding adhesive system but the sixth generation has clinically acceptable shear bond strength. Also, it was found that 6 th generation leaves less residual adhesive on the tooth after bracket removal (ARI score of 3).

\section{REFERENCES}

1. Bond JA, Croll TP. One-step etch/primer for bracket bonding. Pediatr Dent 2001;23(2):145-146.

2. Asgari S, Salas A, English J, Powers J. Clinical evaluation of bond failure rates with new self etching primer. J Clin Orthod 2002;36(12):687-689.

3. Bishara SE, Gordon VV, Vonwald L, Olson ME. Effect of an acidic primer on shear bond strength of orthodontic brackets. Am J Orthod Dentofac Orthop 1998;114(3):243-247.

4. Jorge P. New developments in dental adhesion. Dent Clin N Am 2007;51(2):333-357.

5. Croll TP. Self-etching adhesive system for resin bonding. ASDC J Dent Child 2000 May-Jun;67(3):176-181.

6. Bishara SE, Vonwald L, Laffoon JF, Warren JJ. Effect of a self etch primer/adhesive on the shear bond strength of orthodontic brackets. Am J Orthod Dentofac Orthop 2001; 119(6):621-624.

7. Bishara SE, Ajlouni R, Laffoon JF, Warren JJ. Effect of a fluoride releasing self-etch acidic primer on the shear bond strength of orthodontic brackets. Angle Orthod 2002;72(3):199-202.

8. Kugel G, Ferrari M. The science of bonding from first to sixth generation. J Am Dent Assoc 2000;131(Suppl 1):20S-25S.

9. Frey O. Creating a reliable bond. An all-in-one system. Am J Dent 2000;13spec no.:85D-87D.

10. Miyazaki M, Hinoura K, Ando S, Onose H, Moore BK. Adhesive properties of a newly developed one step bonding system. J Dent Res 2000;79(IADR Abst 1849):375.

11. Breschi L, Perdigao J, Mazzotti G, Nunes MF, Gobbi P. Ultramorphology of self-etching adhesives on ground enamel: a high resolution SEM study. Am J Dent 2003;16 spec no: 57A-62A.

12. Bishara SE, Gordon VV, Von Wald L, Jakobsen JR. Shear bond strength of composite, glass ionomer and acidic primer adhesive systems. Am J Orthod Dentofac Orthop 1999;115(1):24-28.

13. Attar N, Taner Tu, Tulumen E, Kockmaz Y. Shear bond strength of orthodontic brackets bonding using conventional vs one and two step self-etching/adhesive system. Angle Orthod 2007;77(3):518-523.

14. Rueggeberg FA, Fortson WW, Metternbrug DJ. Orthodontic bracket retention strength using a self etching resin primer. J Dent Res 2000;79(IADR Abst. No. 1110):282.

15. Hitmi L, Attal JP, Degrange M. Evaluation of acidic adhesive for orthodontic bonding. J Dent Res 2000;79(IADR Abst. 2906):507.

16. Bergeron C, Vgelinas P, Van Meerbeek B. Bond strength of self-etching adhesive to enamel. J Dent Res 2000;79(IADR Abst. 2386):442.

17. Rogello, Vilchis JS, Yamamoto S, Tal NK, Yamamoto K. Shear bond strength of orthodontic brackets bonded with different self etching adhesives. Am J Orthod Dentofac Orthop 2009;136(3):425-430.

18. Chaki BZ, Kimyou S, Hydari M, Shahbaf S. Effect of selfetching primer/adhesive and conventional bonding on the shear bond strength in metallic and ceramic brackets. Med Oral Patol Cir Bucal 2012;17(1):e164-e170. 